\title{
Design e desenvolvimento de novos produtos através da transferência de conhecimento entre Brasil, Espanha e Portugal
}

\author{
Pacheco, Karla Mazarelo M. ${ }^{\text {; }}$; Paixão-Barradas, Susana ${ }^{\text {b }}$ Silva, Mirella Sousa ${ }^{c}$; Pacheco, Almir de \\ Souza $^{\mathrm{d}}$ \& Nascimento, Claudete Catanhede do ${ }^{\mathrm{e}}$ \\ ${ }^{a}$ Departamento de Design e Expressão Gráfica, Universidade Federal do Amazonas, Manaus-AM/ Brasil - \\ karlamazarelo@hotmail.com \\ ${ }^{b}$ Department of Strategy, Sustainable Development and Entrepreneurship, Kedge Design School, Toulon, Provence- \\ Alpes-Côte d'Azur, France - susana.paixaobarradas@kedgebs.com \\ ${ }^{c}$ Faculdade Martha Falcão - Devry, Manaus, Amazonia, Brazil - mirella.sou@gmail.com \\ ${ }^{\mathrm{d}}$ Faculdade Martha Falcão - Devry, Manaus, Amazonia, Brazil - almirdesigner@gmail.com \\ e Instituto Nacional de Pesquisa do Amazonas (INPA), Manaus, Amazonia, Brazil. ccatanhede@hotmail.com
}

\begin{abstract}
Resumo
Este documento relata a experiência adquirida através de um projeto de cooperação internacional realizado entre quatro instituições de ensino e pesquisa, tendo em vista a aplicação técnica do conhecimento do design para a criação e desenvolvimento de novos produtos a partir do uso de três matérias-primas naturais distintas: fibra, madeira e pedra. Das metas almejadas buscou-se principalmente a capacitação dos discentes de Design do Departamento de Design e Expressão Gráfica da Universidade Federal do Amazonas (DEG/UFAM) para atuação e o desenvolvimento de novos produtos; a valorização de matérias-primas naturais da Amazônia, assim como o saber tradicional, cultural e do design e a troca de conhecimento, tecnologia, experiência profissional e cientifica entre os seus participantes. O projeto foi desenvolvido com base em pesquisas qualitativas e quantitativas, com caráter descritivo, explicativo, exploratório e experimental. Foram realizados: levantamento de dados bibliográficos e de publicações científicas sobre as matérias-primas, pesquisa in loco e estudo de casos. Também realizou reuniões em equipes e aplicou como método de investigação a técnica focus group e a prática de formulários de observação. Como resultados o projeto gerou 13 (treze) protótipos de produtos, com base no conhecimento e técnicas partilhadas entre as instiuições parceiras, 11 (onze) registros de patentes, 1.000 (mil) exemplares de catálogos apresentando as informações técnicas e cientificas referentes aos produtos da pesquisa, e, por fim, fortaleceu as parcerias internacionais e institucionais envolvidas no projeto, com possibilidades de novas ações, em conjunto, para o futuro.
\end{abstract}

Keywords: Design, Matérias-primas naturais, Novos produtos, Transferência de conhecimento, Cooperação Internacional. 


\begin{abstract}
This paper reports the experience gained through an international cooperation project carried out in four educational and research institutions, with a view to technical application of design knowledge for the creation and development of new products from the use of three natural raw materials different: fiber, wood and stone. The desired goals was, primarily, training of Design students of the Department of Design and Graphic Expression of the Federal University of Amazonas (DEG / UFAM) for performance and development of new products; the valuation of natural raw materials from the Amazon, as well as traditional knowledge, cultural and design, and the exchange of knowledge, technology, professional and scientific experience among its participants. The project was developed based on qualitative and quantitative research with descriptive, explanatory, exploratory and experimental. It carried out: survey of bibliographic data and scientific publications about the raw materials, on-site of the research and case studies. Also it held meetings in teams and applied as a research method the focus group technique and practice forms of observation. As a result the project generated thirteen (13) prototypes of products based on knowledge and shared technology between partner institutions, eleven (11) records of patents, one thousand (1,000) copies of catalogs presenting the scientific and technical information relating to products's research, and finally was strengthened the international and institutional partnerships involved in the project, with possibilities of new shares together for the future.
\end{abstract}

Keywords: Design, Natural raw materials, new products, knowledge transfer, international cooperation.

\title{
1. Introdução
}

O desenvolvimento de novos produtos é considerado como um meio importante para a criação e sustentação da competitividade (DE TONI, DEONIR; MILAN, GABRIEL SPERANDIO y SCHULER, MARIA, 2005). Identificar as oportunidades que busquem sistematicamente informações que permitam a organização adequada para a aceitação comercial de produtos e/ou serviços, pode dispor de uma grande probabilidade de sucesso, com soluções às necessidades expressas pelo mercado (PACHECO, K. M. M.; ORTUÑO, B. H.; MIRANDA, I. P. A.; NASCIMENTO, C. C. y PACHECO, A. S., 2011). O potencial existente no uso das matérias primas naturais para o desenvolvimento de novos produtos além de gerar benefícios econômicos à sociedade, também vem sendo cada vez mais valorizado pelos meios acadêmico, científico, tecnológico, e industrial. Universidades, Institutos de Pesquisas e Orgãos de Fomentos, vêem nos recursos naturais a oportunidade de valorizá-los e lhes atribuir novas funções de uso e consumo, através da colaboração mutua entre instituições que prezam pela qualidade da formação e capacitação profissional de pessoas, principalmente aquelas que se preparam, identificam ou atuam no campo de projetos e desenvolvimento de produtos a partir utilização de materias-primas naturais. Sabendo que a formação acadêmica é o primeiro grande passo para que os alunos compreendam como planejar e desenvolver um novo produto, de acordo com as necessidades do mercado, agregando a isso fatores estratégicos e inovadores que possam garantir a sua aceitação por parte dos consumidores, e que a 
transferência de conhecimento, de tecnologia e de experiencia são aspectos primordiais durante esse processso, o Departamento de Design e Expressão Gráfica ((DEG) da Universidade Federal do Amazonas (UFAM/Manaus-Brasil); o Laboratório de Engenharia de Artefatos de Madeira (LEAM) do Instituto Nacional de Pesquisas da Amazônia (INPA/Manaus - Brasil); o Departamento de Ingeniería Gráfica del Diseño (DIG) da Universitat Politècnica de València (UPV/Valencia - Espanha) e o Centro Tecnológico da Pedra Natural de Portugal (CEVALOR/Borba - Portugal), uniram-se através do apoio financeiro da Coordenação de Aperfeiçoamento de Pessoal de Nível Superior (CAPES/ Brasil), para desenvolver um Projeto de Cooperação Interncional, intitulado como: Design e Desenvolvimento de Novos Produtos a Partir de Matérias-Primas Naturais da Amazônia, que colocasse em prática a aplicação técnica do conhecimento do design para a criação e desenvolvimento de novos produtos a partir do uso de três matérias-primas naturais distintas: fibra, madeira e pedra. $\mathrm{O}$ trabalho também contemplou os seguintes objetivos:

- Valorizar as matérias-primas naturais da Amazônia, assim como o saber tradicional, cultural e do design;

- Trocar conhecimento, tecnologia, experiência profissional e científica entre os seus participantes;

- Capacitar os discentes de Design do Departamento de Design e Expressão Gráfica da Universidade Federal do Amazonas (DEG/UFAM) para atuação e o desenvolvimento de novos produtos;

\section{A proposta do Projeto}

A ideia para desenvolver um projeto de cooperação internacional, partiu da tentativa de relacionar e unir elementos naturais de lugares distintos para a composição estrutural de um design de produto. Com essa intenção, a proposta buscou envolver a participação de três países: Brasil, Espanha e Portugal, e com eles trabalhar uma parceria em prol do uso de matérias-primas naturais, entre elas: da Amazônia e da Europa, para a gestão e fabricação de novos produtos, de modo a valorizar e prezar pelos aspectos técnicos, científicos, socioculturais, econômicos e eco sustentáveis. Outro fator também a ser considerado para a prática da pesquisa, seria ter o design como agente gerenciador das atividades e dos processos de transformação desses recursos naturais e de outros materiais que pudessem ser necessários para a criação e o desenvolvimento dos produtos. Para a materialização da proposta foi importante identificar as instituições que poderiam fazer parte da equipe técnica do projeto e com elas definir quais as matériasprimas a serem investigadas, os objetivos que deveriam ser alcançados e as etapas necessárias para a realização da pesquisa. Os convites para compor parcerias foram feitos às seguintes instituições de ensino, pesquisa e tecnologia: Instituto Nacional de Pesquisas da Amazônia (INPA- Brasil); Universitat Politècnica de València (UPV - Espanha); e Centro Tecnológico da Pedra Natural de Portugal (CEVALOR - Portugal), que após conhecerem melhor os princípios do trabalho, aceitaram de imediato a fazer parte do mesmo. A Universidade Federal do Amazonas (UFAM), através do Departamente de Design e Expressão Gráfica - considerado o autor da proposta, foi nomeada a sede do projeto. A iniciativa obteve a aprovação por parte da Coordenação de Aperfeiçoamento de Pessoal de Nível Superior (CAPES), que financiou a realização da pesquisa no Brasil, por meio do Programa Pesquisador Visitante Especial (PVE), conforme a Chamada Pública ํㅜ 61/2011. 
Design e desenvolvimento de novos produtos através de transferência de conhecimento entre Brasil, Espanha e Portugal.

\subsection{Objetos do estudo}

A opção de trabalhar com a fibra vegetal de tucumã-i (Astrocaryum acaule) do Amazonas, algumas espécies de madeiras naturais da Amazônia e a pedra mármore branco de Portugal com veios, como objetos de estudo, foi realizada em concordância com a Coordenadora Técnica do Projeto, a Pesquisadora Visitante Especial e a CAPES e também em função das especialidades e produções científicas das pesquisadoras principais e das instituições parceiras que dispunham das referidas matérias-primas. Os recursos naturais utilizados na proposta possuem características técnicas interessantes. E quando adaptados para produtos são capazes de oferever muitas vantagens aos consumidores e ao mercado. A seguir, alguns dos dados identificados e considerados importantes em cada um deles.

\subsubsection{Fibra de tucumã-i (Astrocaryum acaule)}

Esta fibra deriva do tucum originada pelo gênero Astrocaryum da espécie acaule, também conhecida por tucumã-i (Fig.1), uma classe de palmeira localizada em grande parte na região do Alto Rio Negro - no estado do Amazonas, norte do Brasil. É produzida, cultivada e processada de forma sustentável e artesanal por comunidades indígenas e caboclas (MACIEL et al., 2011). Possui características físicas plausíveis, e pode ser aplicada em diversos tipos de produtos, desde acessórios para uso pessoal como pulseiras, colares e bijuterias, até produtos com uma escala maior, como vasos, cestos e até mesmo roupas. O uso da fibra em produtos é flexível, pois é útil para diversas categorias (PACHECO et al.,2011). Além da aparência exótica e o aspecto visual atrativo, é bastante resistente, sem produzir elementos tóxicos ou mal cheiro, possibilitando uma aplicação segura, reforçada pela maleabilidade da sua estrutura que torna-se adaptável aos mais diferentes e variados tipos de produtos.
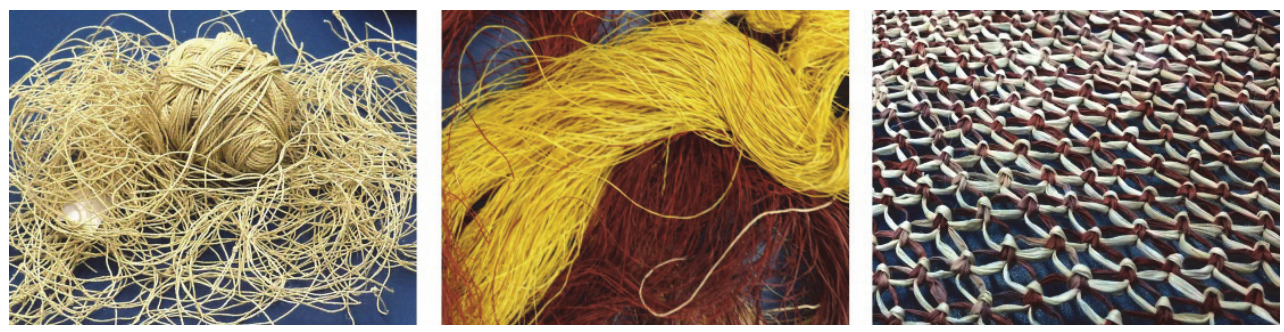

Fig. 1 Fibra de tucumã-i (Astrocaryum acaule) e sua aparencia exótica

\subsubsection{Pedra mármore branco de Portugal com veios}

A pedra mármore branco de Portugal com veios (Fig.2), apresenta como características inerentes: dureza, resistência e vergadas. Descrevendo-a macroscopicamente tem-se: calcário microcristalino rosado, bioclástico a bioconstruído, abundantemente fossilífero, com bastantes estilólitos, alguns deles semiabertos, e esparitizado (INETI, 2014). O seu processo de produção envolve a mistura de alta tecnologia com o trabalho artesanal. A sua aplicação e funcionalidade são amplamente justificadas pelas suas características técnicas. Além de apresentar boas características físicas e mecânicas, resistência e durabilidade, observando o comportamento humano a partir de produtos ou ambientes criados a partir dessa matéria-prima. Além disso, possui em suas características intrínsecas, que influenciam o bem-estar humano, os fatores-chave do material na criação de novos produtos, assim como muitas outras variáveis consideradas importante a todo o processo (CEVALOR, 2014). 

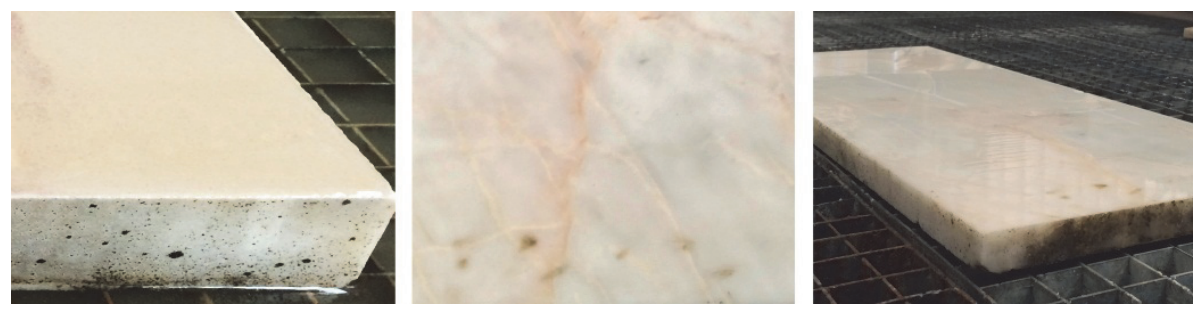

Fig. 2 Pedra mármore branco de Portugal com veios

\subsubsection{Madeiras naturais da Amazônia}

As madeiras amazônicas (Fig.3) por sua facilidade de obtenção e manuseio, são um dos recursos naturais mais utilizados pelo homem, os produtos gerados a partir delas atendem satisfatoriamente diversos tipos de mercados e consumidores, por apresentarem boa durabilidade e adaptação ao processo de acabamento, alta resistência física e mecânica, variações entre alta e média densidade (NASCIMENTO y MONTEIRO DE PAULA, 2012).
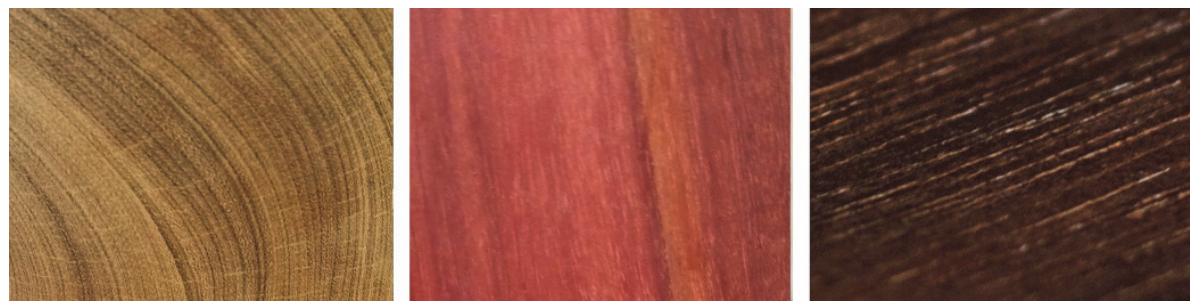

Fig. 3 Algumas das madeiras amazônicas utilizadas no projeto

Devido a grande variedade, é comum que se atribua o mesmo nome vulgar às várias espécies de madeiras botanicamente distintas, e do mesmo modo, com propriedades tecnológicas diferenciadas. Ainda que estas matérias-primas gerem resíduos a partir dos seus processos de adaptação para produtos, estes mesmos resíduos podem ser reaproveitados e transformados em novos produtos, atividade que condiz com o propósito da eco sustentabilidade.

Assim, das madeiras amazônicas selecionadas para o projeto destacam-se: itaúba (Guarea trichilioides L.), maçaranduba (Manilkara huberi Ducke Satnd), cedrinho (Erisma uncinatum), tanibuca (Buchenavia huberi Ducke), angelim-pedra (Hymenolobium petraeum Ducke), marupá (Simarouba amara Aubl), louro Aritu (Licaria aritu Ducke), guariúba (Claricia raecemosa Ruiz), sucupira (Bowdichia nitida), cedro (Cedrela fissilis) e angelim-vermelho (Andira parviflora Ducke).

\subsection{Participação das instituições parceiras no projeto}

O projeto foi estruturado com base em quatro vertentes: Ensino, Pesquisa, Tecnologia e Mercado, visando também os aspectos científicos, socioculturais, econômicos e eco sustentáveis. Para tanto, a parceria realizada com duas instituições europeias (Espanha e Portugal), uma local (pertencente à cidade de Manaus no estado do Amazonas/Brasil) e uma de fomento de caráter nacional (Brasil), foi extremamente importante e estratégica. Cada instituição envolvida (Fig.4), pôde colaborar de acordo com a sua expertise e proporcionar espaços, equipe técnica e materiais para a realização da pesquisa. 

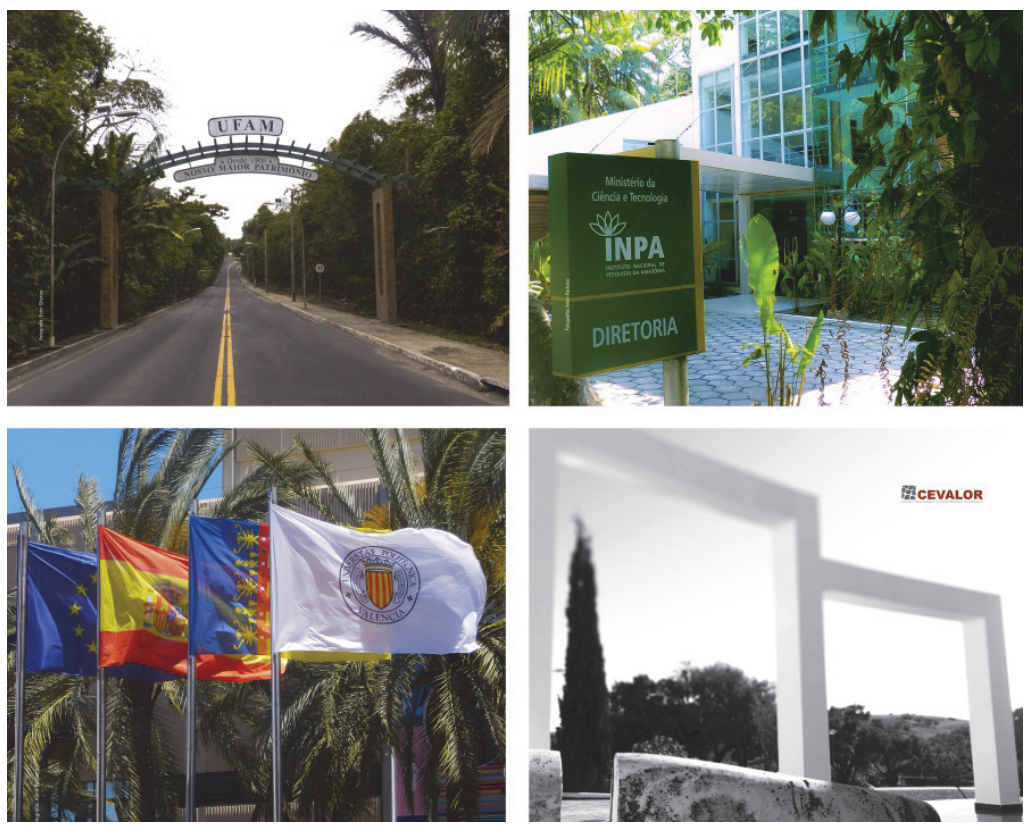

Fig. 4 Instituições parceiras do projeto: UFAM, INPA, UPV e CEVALOR

Das competências atribuídas e prestadas por cada instituição parceira:

- Coordenação de Aperfeiçoamento de Pessoal de Nível Superior (CAPES/ Brasil) - Orgão financiador do projeto e investidor na formação e aperfeiçamento de recursos humanos e de alto nível no país e exterior, serve de instrumento para a comunidade universitária na busca de um padrão de excelência acadêmica para prática e desenvolvimento de projetos e pesquisas e promoção da cooperação científica internacional;

- Departamento de Design e Expressão Gráfica (DEG) da Universidade Federal do Amazonas (UFAM/Manaus-Brasil) - Sede de desenvolvimento do projeto. Como coordenação geral da pesquisa, disponibilizou 01 (um) laboratório de design: para a prática das atividades de produção de relatórios técnicos, reuniões com as equipes de trabalho, realização de briefings e demais métodos de investigação, criação das alternativas para protótipos e acompanhamento de trabalhos manuais concretizados pelos especialistas técnicos e artesãs participantes da equipe; e 01 (uma) marcenaria: para a produção de uma parte das peças idealizadas pelo projeto. O DEG foi responsável por liderar as investigações feitas sobre a fibra de tucumã-i (Astrocaryum acaule), e orientar quanto a postura e conhecimento do design a serem adotados e aplicados durante às ações tomadas para o levantamento e análise de dados, definição dos requisitos e parâmetros para os processos de criação e geração das alternativas de protótipos, produção e acabamento dos protótipos, criação e desenvolvimento do material gráfico e de divulgação do projeto, planejamento e organização das exposições dos produtos e produção de relatório final com os resultados do projeto;

- Laboratório de Engenharia de Artefatos de Madeira (LEAM) do Instituto Nacional de Pesquisas da Amazônia (INPA/Manaus - Brasil), parceiro e colaborador no processo de investigação e escolha das espécies de madeiras amazônicas utilizadas pelo projeto. O LEAM foi responsável por liderar as investigações feitas sobre as madeiras amazônicas, orientou quanto os aspectos tecnológicos, processos de adaptação e produção dos produtos a partir delas. Assessorou 
tecnicamente na construção das informações, sobre as espeficie catalogadas e certificadas, para esclarecer a respeito do uso das madeiras nas composições estruturais dos produtos. Auxiliou a equipe técnica do projeto durante as pesquisas de campo realizadas em cidades que disponham de produtos artesanais desenvolvidos com as mesmas madeiras utilizadas no projeto. Disponibilizou mais de 15 (quinze) espécies de madeira amazônica para a realização do projeto, 01(um) laboratorio técnico equipado com ferramentas e maquinários para proceder com a fase de levantamento de dados sobre as madeiras e 01 (uma) marcenaria para a produção da outra parte das peças idealizadas pelo projeto;

- Departamento de Ingeniería Gráfica del Diseño (DIG) da Universitat Politècnica de València (UPV/Valencia - Espanha), parceiro e colaborador no processo de orientação sobre as atividades técnicas do design. O DIG, em conjunto com o DEG, forneceu informações técnicas sobre os atributos do design necessários para o desenvolvimento de novos produtos e sobre os métodos sistêmicos a serem aplicados durante as etapas de criação e geração de alternativas para os protótipos. Recepcionou a Pesquisadora Visitante Especial, nas dependências da Escuela Técnica Superior de Ingeniería del Diseño, para junto desenvolverem um workshop sobre as pesquisas levantadas no Brasil e Portugal, referentes aos objetos de estudo, e acompanharam as criações (propostas de produtos) dos alunos de design da UPV para avaliarem e decidirem os protótipos do DIG a serem confeccionados pela equipe técnica do projeto nas dependências do DEG/UFAM e LEAM/INPA ambos no Brasil, como também no Centro Tecnológico da Pedra Natural de Portugal CEVALOR, em Borba/Portugal.

- Centro Tecnológico da Pedra Natural de Portugal CEVALOR/Borba - Portugal), parceiro e colaborador no processo de adaptação e uso da pedra mármore branco de Portugal com veios para o desenvolvimento dos produtos do projeto. A CEVALOR juntamente com a Pesquisadora Visitante Especial, foram as fontes de orientações e informações sobre a pedra - um dos objetos de estudo. $\mathrm{O}$ centro disponibilizou a sua marmoraria com maquinário eficiente e de primeira linha, e 01 (um) técnico profissional para assessorar a equipe técnica do projeto nos trabalhos de confecção dos protótipos que possuíam a pedra mármore branco de Portugal com veios em suas estruturas. Relacionou as atividades de investigação sobre a pedra com a importância da transferência tecnológica, demonstrando as etapas de adaptação e transformação da mesma, ressaltando o bom uso da matéria-prima, formando o conhecimento e informando o diferencial das rochas ornamentais e industriais.

\subsection{Formação, capacitação, troca de conhecimentos, experiências e tecnologias}

O projeto inicialmente buscou preparar o conhecimento dos seus participante sobre a importância do uso de matérias-primas naturais da amazônia junto a um recurso natural europeo (pedra), para o desenvolvimento de novos produtos. Para isso, a equipe técnica do projeto (Fig.5), dividiu-se em três grupos distintos: grupo de pesquisa sobre a fibra de tucumã-i (Astrocaryum acaule); grupo de pesquisa sobre as madeiras naturais da Amazônia; e grupo de pesquisa sobre a pedra mármore branco de Portugal com veios. Após a referida divisão, foi dado inicio a fase do levantamento de dados sobre cada objeto de estudo. 
Design e desenvolvimento de novos produtos através de transferência de conhecimento entre Brasil, Espanha e Portugal.
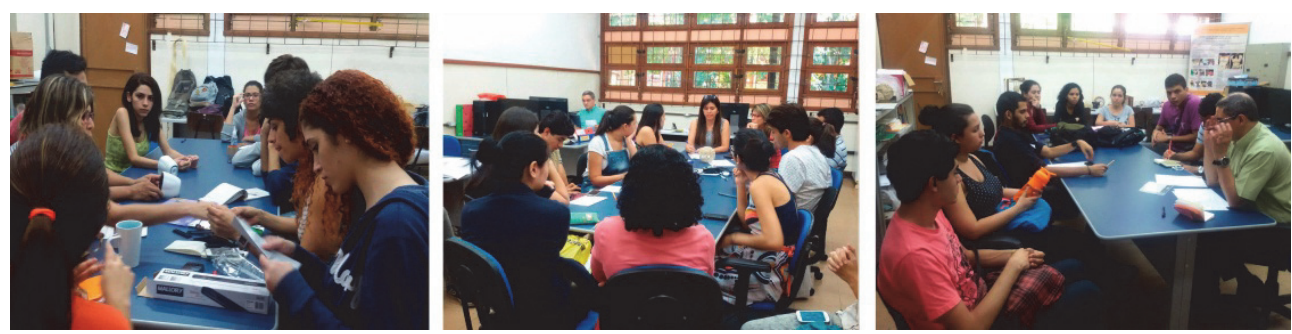

Fig. 5 Equipe técnica do projeto em reunião para divisão dos 3 grupos de pesquisa distintos

Cada equipe (Fig.6, 7 e 8), recebeu as devidas orientações por parte de suas coordenadoras de pesquisa, e saíam a campo em busca de informações técnicas e científicas, assim como exemplos de experimentos ou estudos similares que tenham registrado dados sobre tais recursos. Os componentes de cada grupo tiveram a oportunidade de consultar, dialogar, trocar e registrar informações a respeito do objeto da sua pesquisa junto a especialistas, instituições, lojas, empresas e mercado que trabalham com o tema da investigação.
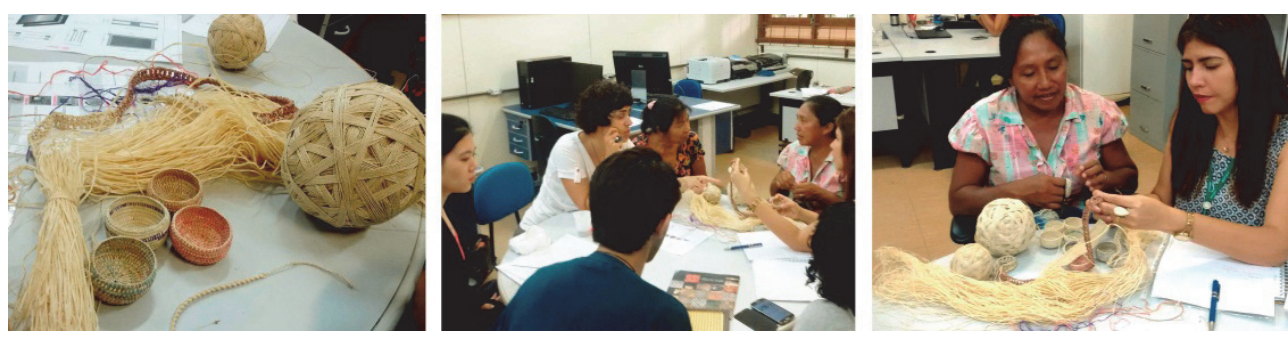

Fig. 6 Grupo de pesquisa sobre a fibra de tucumã-i (Astrocaryum acaule)
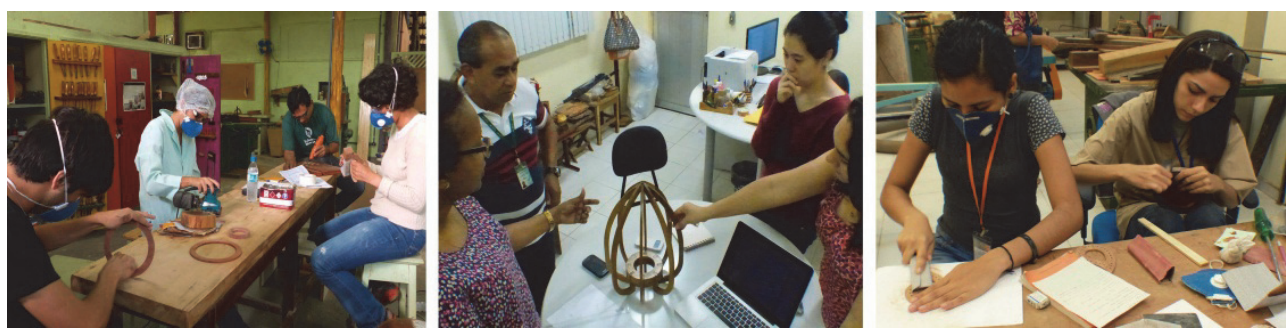

Fig. 7 Grupo de pesquisa sobre as madeiras naturais da Amazônia
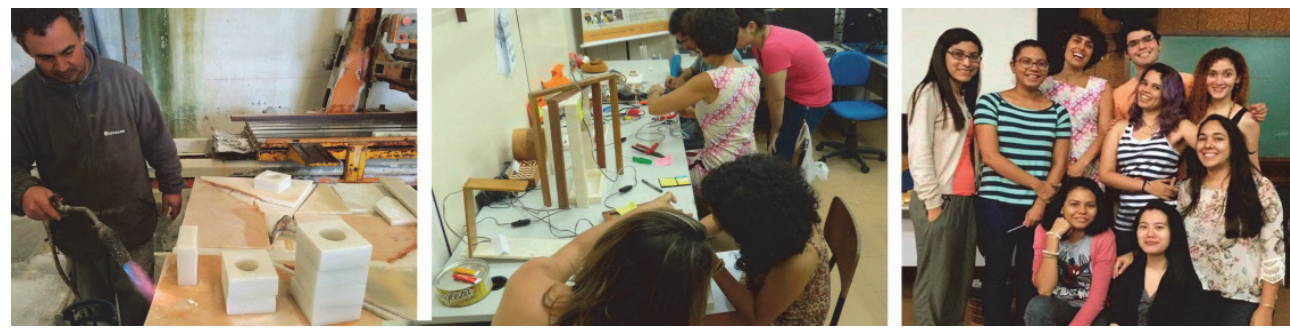

Fig. 8 Grupo de pesquisa sobre a pedra mármore branco de Portugal com veios 
Esses contatos, puderam relatar, mostrar e indicar a todos os participantes do projeto as oportunidades e limitações que as materiais primas poderiam apresentar com respeito ao proposito da pesquisa. Ao passo que cada grupo conseguia ir compreendendo o universo do seu objeto de estudo, produzia um relatório parcial resumindo os dados capturados a cada etapa da investigação. Tais documentos tornavam-se a bússola que direcionava os caminhos a serem percorridos mais a diante. É importante ressaltar que, a cada relatório parcial entregue, os três grupos se reuniam e cada um deles explanava o que haviam vivenciado e aprendido até aquele momento. Durante as explanações, os representantes das instituições parceiras e unidade sede do projeto faziam também as suas contribuições, de modo a ajudar às equipes nas tomadas de decisões e futuras ações para o andamento das pesquisas. Esta primeira experiência (divisão das equipes e o levantamento de dados sobre os objetos de estudo) proporcionou aos discentes, docentes, técnicos e colaboradores do projeto um aprendizado em conjunto, o que facilitou as trocas de informações e definição dos métodos e as atividades que poderiam ser utilizadas para registrar dados e melhor aproveitá-los durante as fases da criação, desenvolvimento e produção dos protótipos da pesquisa.

\subsection{Proposta para novos produtos}

Uma vez compreendido o universo de dados levantados sobre cada recurso natural estudado, as coordenadoras das equipes reuniram-se para definir os requisitos e parâmetros que deveriam ser trabalhados durante a etapa da geração de alternativas para a confecção dos protótipos dos produtos. Das condições estabelecidas, foi determinado que os produtos elaborados deveriam atender a ambientes comerciais de alto nível, como: hotéis, restaurantes e demais estabelecimentos com enfoques temáticos. $\mathrm{O}$ projeto do produto deveria contemplar em sua estrutura, no mínimo, duas das matérias primas estudadas, com o propósito de comparar a evolução e/ou o enriquecimento material e visual das peças. Dado o desafio, cada aluno deu inicio a criação da sua proposta de produto. Ainda que as propostas fossem individuais, os grupos faziam questão de se reunir e discutir sobre suas ideias (Fig 9).
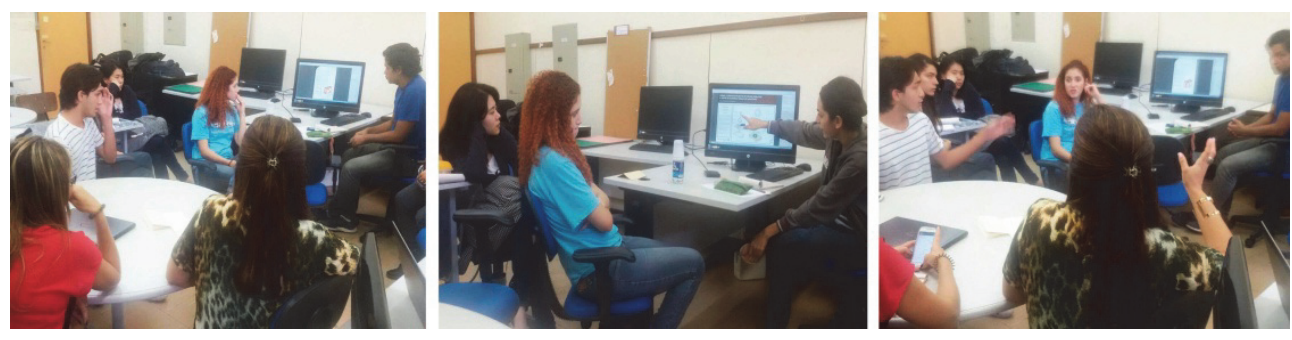

Fig. 9 Alunos e professores do projeto, reunindo e discutindo ideias para a geração de alternativas

Da mesma forma, faziam questão de se aconselhar com os representantes das instuições parceiras através de consulta local, e-mail ou vídeo, para saber o que pensavam a respeito de suas criações (Fig.10), o interesse pelas opiniões eram primordiais quando partiam das universidades, institutos e empresas colaboradoras que trabalhavam diretamente com as matérias-primas, as quais os alunos queriam utilizar em suas propostas. Mais uma vez, a troca de conhecimento e informações foi fundamental durante esta fase, inclusive com demonstrações de algumas tecnologias e ferramentas de trabalho (pertencentes a essas instituições) que poderiam axiliar na materialização das ideias. Assim, a cada orientação dada, os alunos melhoravam os conceitos sobre suas propostas e trabalhavam com mais representação os seus desenhos. 

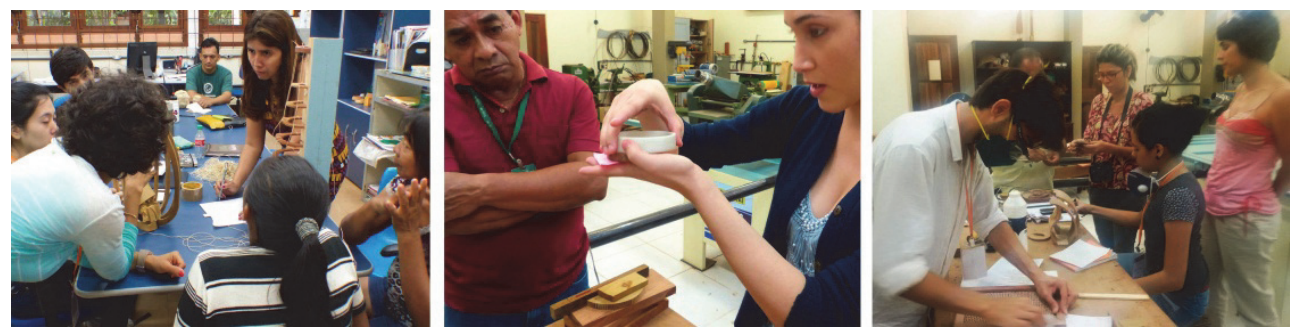

Fig. 10 Alunos discutindo suas ideias com colaboradores de instituições parceiras do projeto

É importante destacar que a geração de alternativas foi realizada tanto pelos alunos de graduação de design da Universidade Federal do Amazonas (DEG/UFAM) como também pelos alunos de pósgraduação (mestrado) da Universitat Politècnica de Valencia (DIG/UPV) na Espanha. Estes últimos, tiveram a oportunidade de participar de um workshop realizado pela Professora Visitante Especial do projeto, na cidade de Valencia/Espanha, que relatou e apresentou os resultados da fase de levantamento de dados realizados, meses antes, pelos grupos de pesquisas formados pelos alunos de design da UFAM. As atividades relacionadas à geração de alternativas resultou na produção de painéis semânticos (Fig.11 e 12), originados pelos alunos da UFAM e da UPV, com base nos requisitos e parâmetros estabelecidos e explicações técnicas e conceituais do design à materialização das suas propostas de produtos.

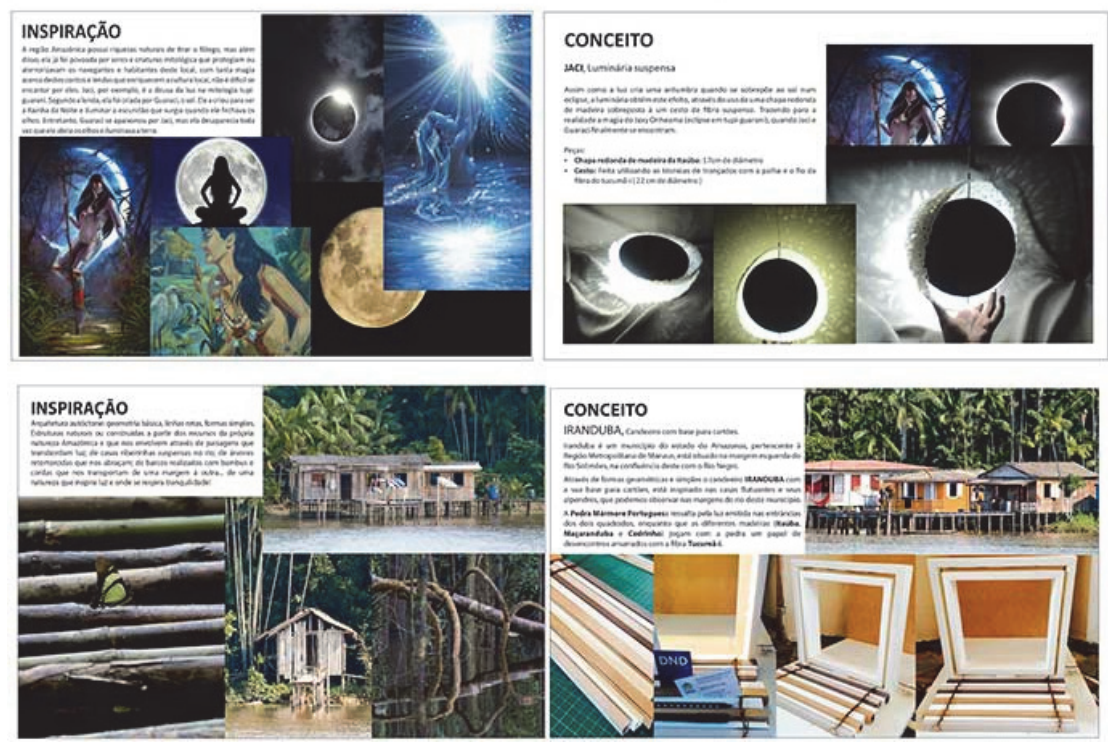

Fig. 11 Exemplo de painéis semânticos gerados pelos alunos participantes do projeto 


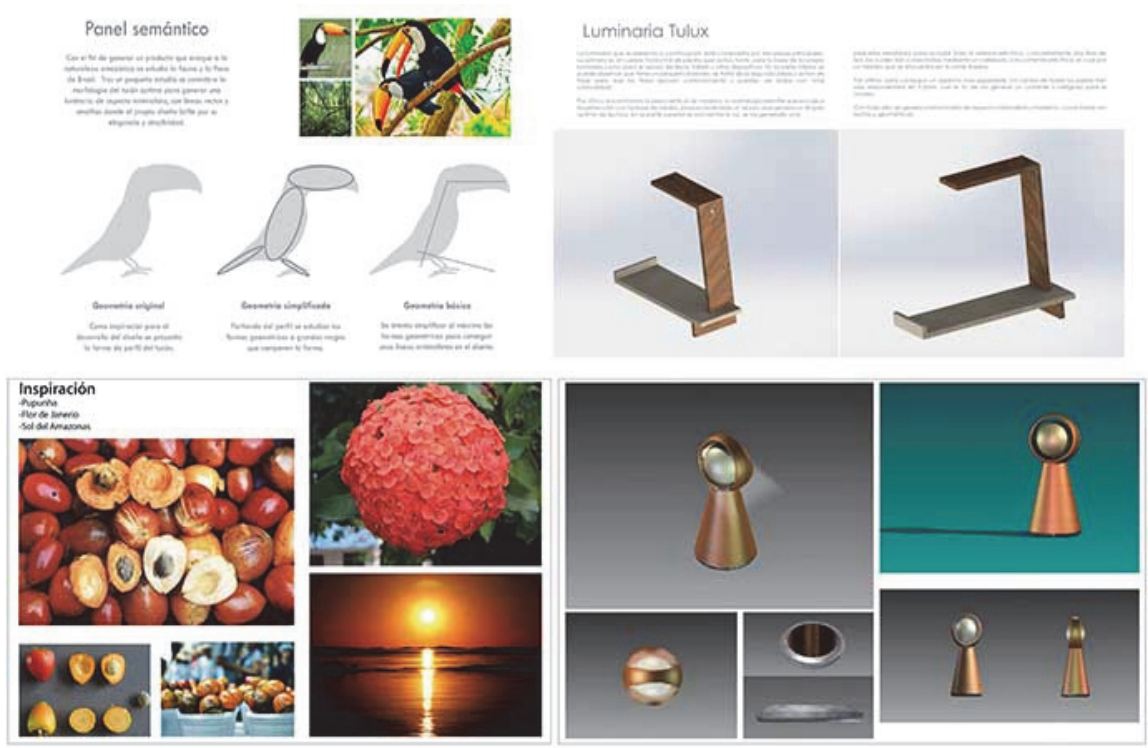

Fig. 12 Outro exemplo de painéis semânticos gerados pelos alunos participantes do projeto

As propostas geradas pelos alunos também foram inspiradas em elementos da fauna e da flora da região amazônica, o que pode ser considerado um grande enriquecimento cultural para todos os participantes, além de valorizar ainda mais a estruturação física e estética dos produtos.

\section{Materiais e métodos}

A pesquisa teve como propósito trabalhar com dados qualitativos e quantitativos, classificados como: exploratórios, descritivos, explicativos e experimental (Gil, 2010). Apresenta uma abordagem conceitual sobre as percepções das pessoas sobre as questões sociais e fatos sobre o estado atual do fenômeno em estudo, descrevendo a natureza das condições em uma situação (Marconi e Lakatos, 2006). Um breve estudo estatístico, cujos dados foram organizados e analisados a partir da distribuição de freqüência e o cálculo de médias, permitiu observar as opiniões coletadas, o problema e o tipo de impacto entre os seus elementos (Blaxter, Hughes, \& Tight, 2002), de modo a determinar a força da associação ou correlação entre as variáveis resultantes da opnião de uma população específica. Como fontes de investigação foram utilizados: livros, artigos publicados em congressos e revistas científicas, registros de entrevistas, jornais e dados divulgados por instituições ou pesquisadores, em págnias de internet. O universo da pesquisa contemplou três grupos de participantes distintos: Proprietários e/ou vendedores de estabelecimentos de comercialização dos recursos naturais investigados, na cidade de Manaus; comunidade acadêmica do Departamento de Design e Expressão Gráfica da Universidade Federal do Amazonas, também pertencente à cidade de Manaus/Amazonas - Brasil; e comunidade acadêmica do Departamento de Ingeniería Gráfica da Escuela Tecnica Superior de Ingeniería de Diseño da Universitat Politècnica de València, na cidade de Valência/Espanha. O tempo para a realização do referido trabalho foi de um ano e meio e dos procedimentos adotados para a coleta de informações, ferramentas e métodos para o controle e qualidade dos dados, a equipe do projeto utilizou: formulários de orientação - produzidos pelas coordenadoras de cada grupo de trabalho específico, para o registro da pesquisa de campo realizada junto ao mercado de produtos naturais; reuniões semanais com os grupos de pesquisa (focus group) para explanação e discussão sobre os dados coletados - tais reuniões eram registradas com câmera fotográfica, filmadora e os resultados gravados em arquivos digitais para posterior produção do relatório final do 
projeto; questionário de observado, produzido pela coordenação geral do projeto e a pesqusiadora visitante especial, para o registro das informações capturadas durante as exposições dos produtos da pesquisa. Para o processo de criação das propostas, os alunos utilizaram das técnicas conceituais do desenho técnico, de observação, renderização, construções de painéis semânticos. Dos softwares gráficos utilizados foram: CorelDraw, Illustrator, Indesign e Photoshop. E dos softwares 3D: AutoCAD, 3D Max, Blender, etc. O processo de confecção das peças foi realizado nos laboratórios, marcenarias e marmoraría das 4 (quatro) instituições parceiras - Brasil, Espanha e Portugal, com assessoramento constante dos seus pesquisadores, docentes e técnicos. Os recursos naturais de estudos foram doados, em forma de resíduos, pelo Instituto Nacional de Pesquisas da Amazônia (INPA) - doador da madeira, Centro Tecnológico da Pedra Natural de Portugal (CEVALOR) - doador da pedra, e a empresa de artesanato Arte Tukano pertencente a duas artesãs colaboradoras do projeto, que são vinculadas à comunidade Juquira produtora da fibra de tucumã-i (Astrocaryum acaule), no Amazonas. O projeto também realizadou 2 (duas) exposições, para as quais desevolveram-se os materiais gráficos de divulgação dos resultados e a produção de um catálogo visual com as informações técnicas e conceituais do projeto e, consequentemente, dos seus produtos - os quais foram reagistrados para obtenção das patentes junto à Pro-reitoria de Inovação Tecnológica da Universidade Federal do Amazonas (UFAM) no final do ano de 2015.

\section{Resultados}

O processo de confecção dos produtos foi árduo, trabalhoso e intenso. Todas as práticas foram acompanhadas tanto pelas coordenadoras das equipes e colaboradores parceiros, como por cada autor das peças. Para cada dia de produção dos protótipos eram registrados os dados da confecção. Para o processo de produção das propostas geradas, as atividades distribuíram-se entre o Laboratório de Artefatos de Madeira - LEAM, do Instituto Nacional de Pesquisas da Amazônia, e a Marcenaria do curso de Design da Universidade Federal do Amazonas, ambos dotados de maquinários, equipamentos e técnicos especializados para os procedimentos operacionais necessários à produção das peças. Os treze produtos selecionados tiveram como critério de seleção principal, a viabilidade de produção. Analisadas as formas de confecção de cada produto selecionado, foi dado início ao processo de produção dos mesmos. Os primeiros produtos confeccionados, reuniam em sua estrutura os recursos naturais de pedra mármore branco de Portugal com veios e de madeira. Assim sendo, as peças que exigiam a pedra na sua formação tiveram estas partes trabalhadas no Centro Tecnológico da Pedra Natural de Portugal (Fig. 13), na cidade de Borba em Portugal, sob a supervisão da Pesquisadora Visitante Especial e de um técnico profissional da CEVALOR. Quando as peças não apresentavam a estrutura ideial e conforme os desenhos técnicos dos alunos, o técnico e a pesquisadora verificavam as possibilidades de eventuais modificações e trabalhavam tais mudanças de acordo com o consentimento dos autores.

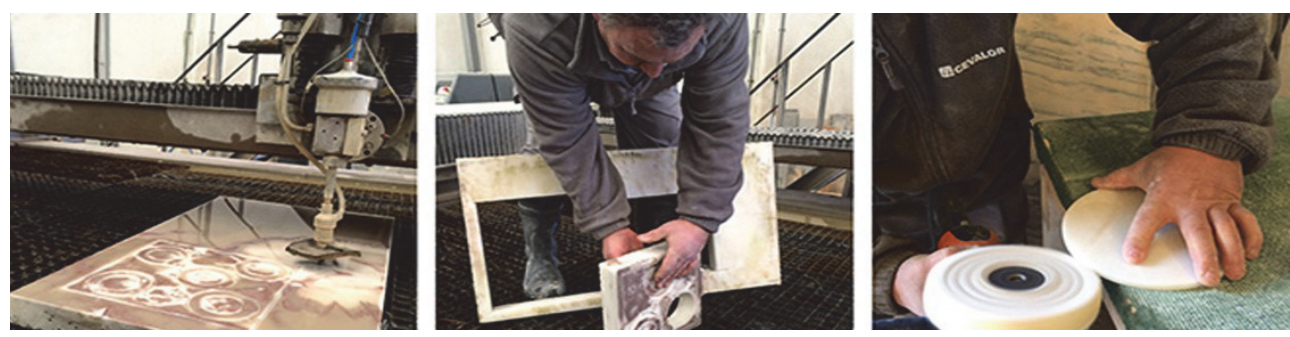

Fig. 13 Processo de produção de um dos produtos com a pedra mármore branco de Portugal com veios 
Após confeccionadas todas as partes de peças que exigiam conter a pedra em suas estruturas, foi iniciado a produção dos produtos com a fibra de tucumã-i (Astrocaryum acaule), e com as madeiras amazônicas. O trabalho realizado com a fibra foi realizado no Laboratório de Design, do Departamento de Design e Expressão Gráfica da Universidade Federal do Amazonas e procedido por duas artesãs indígenas (Fig. 14), que junto a alunos e professores, fizeram a escolha dos tipos de tramas e as cores da fibra.
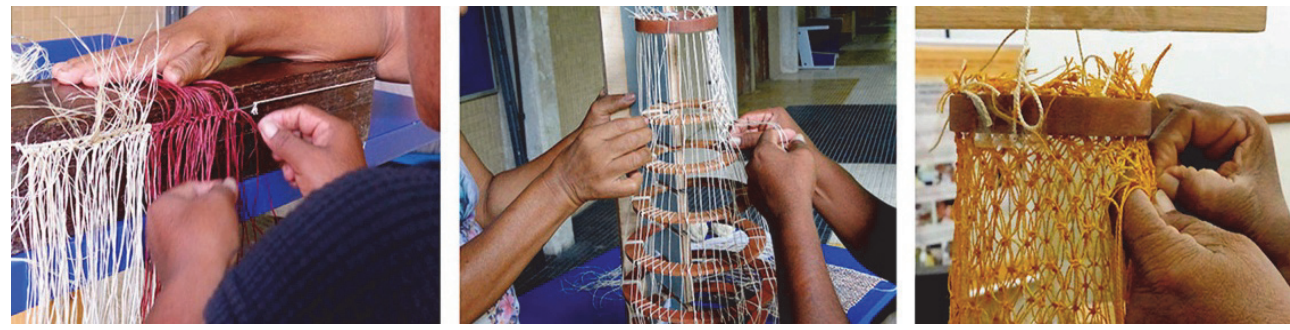

Fig. 14 Processo de confecção de produtos com a fibra de tucumãi (Astrocaryum acaule)

Como muitos dos produtos, embora utilizassem fibra na proposta, não possuíam o detalhamento de como as tramas de composição dos tecidos, gerados a partir da fibra, poderiam ser fixadas nos produtos, permaneceu-se na realização de ajustes e modificações ao longo da execução dos projetos, fomentando a troca de experiências entre alunos, professores e artesãs. As partes dos produtos com madeira (Fig. 15), também foram executadas paralelamente ao trabalho feito com a fibra, sendo que apresentando maior complexidade. Todo o processo foi realizado tanto no Laboratório de Engenharia de Artefatos de Madeira do Instituto Nacional de Pesquisas da Amazônia (LEAM/INPA), uma vez que este laboratório além de possuir maquinário mais atual, também é referência em tecnologia da madeira, como na marcenaria do DEG na UFAM. A princípio as partes projetadas em madeira foram confeccionadas em materiais derivados como tábuas de madeira compensada, pois as espécies doadas para o projeto ainda não haviam chegados na ocasião. Após esse primeiro experimento e a doação das madeiras, a confecção foi orientada conforme os desenhos técnicos dos alunos e conhecimento dos especialistas envolvidos.
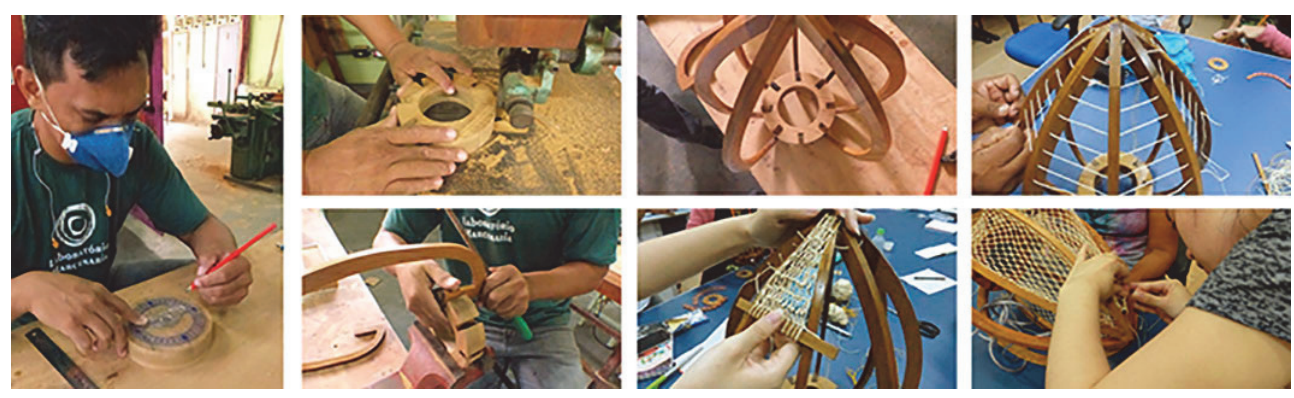

Fig. 15 Processo de confecção de produtos com madeiras amazônicas

Finalizada a etapa de confecção dos produtos, a equipe técnica do projeto realizou o registro fotográfico das peças nos ambientes idealizados para o uso das mesmas (Fig.16). 

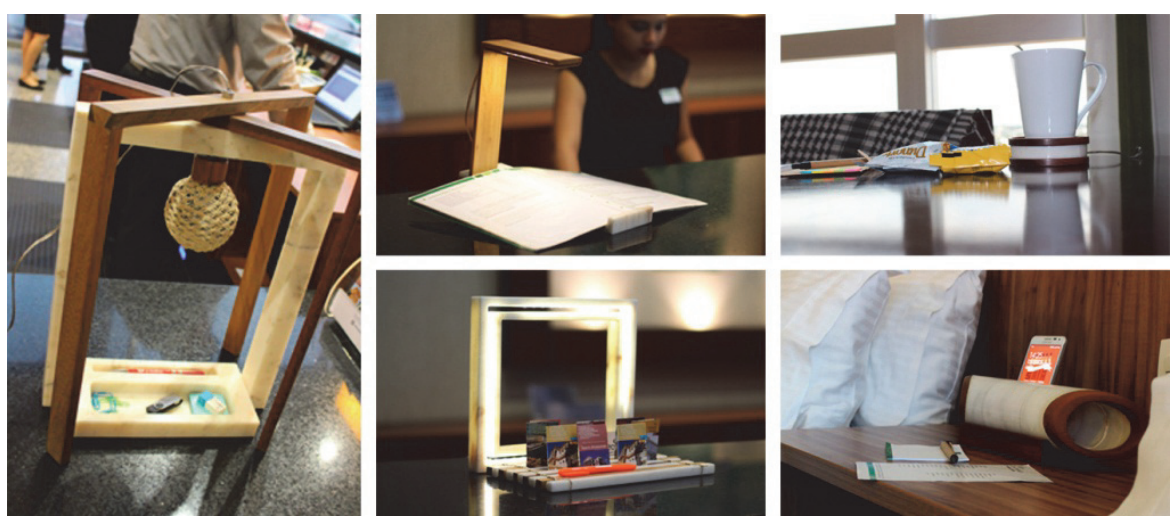

Fig. 16 Registro fotográfico de alguns dos produtos em ambientes de hotéis e restaurantes temáticos

Em paralelo a referida prática, teve inicio a produção de informações técnicas para a elaboração do Catálogo Visual dos produtos e também do material de divulgação do projeto. Durante registro fotográfico, muitos curiosos perguntaram sobre a origem dos produtos e como ter acesso a eles através da venda e comercialização no mercado. Naquele momento, não foi dada nenhuma informação a respeito, uma vez que os alunos sabiam que não era possível divulgar dados da investigação e concepção dos produtos, devido os mesmos estarem em processo de tramitação para registro de patentes na UFAM. No entanto, como parte dos resultados alcançados pelo projeto, a Pro-reitoria de Inovação Tecnológica (PROTEC/UFAM) permitiu que a equipe técnica apresentasse os trezes produtos gerados, nas exposições (Fig. 17 e 18), programadas para as comunidades acadêmicas do DEG/UFAM, no Brasil e do DIG/ETSID, na Espanha.

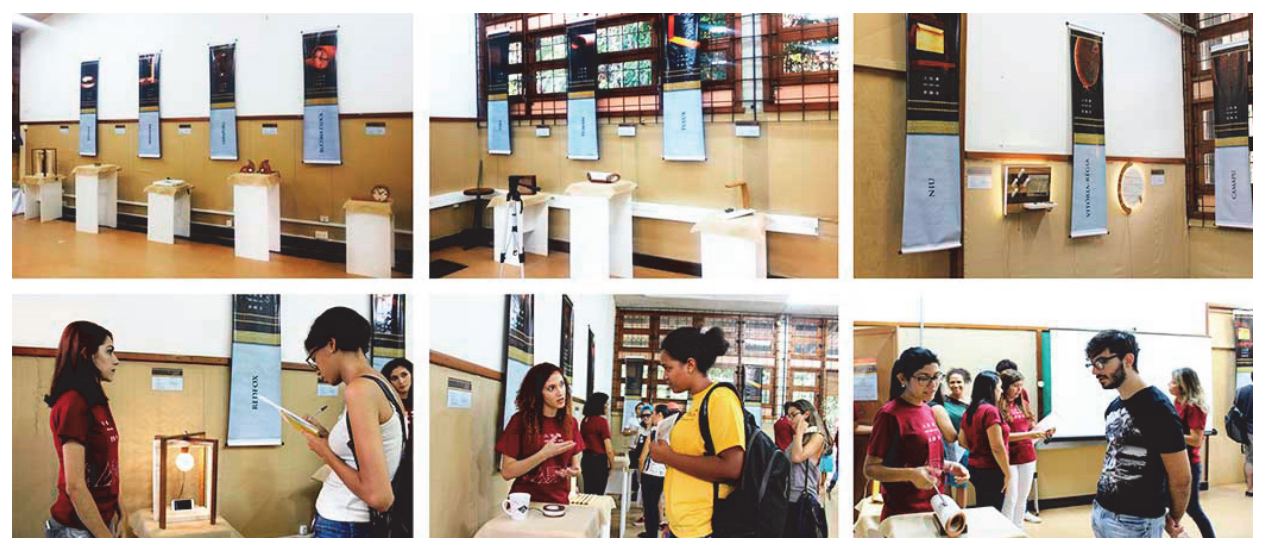

Fig. 17 Exposição dos protótipos na Universidade Federal do Amazonas (DEG/UFAM - Brasil) 

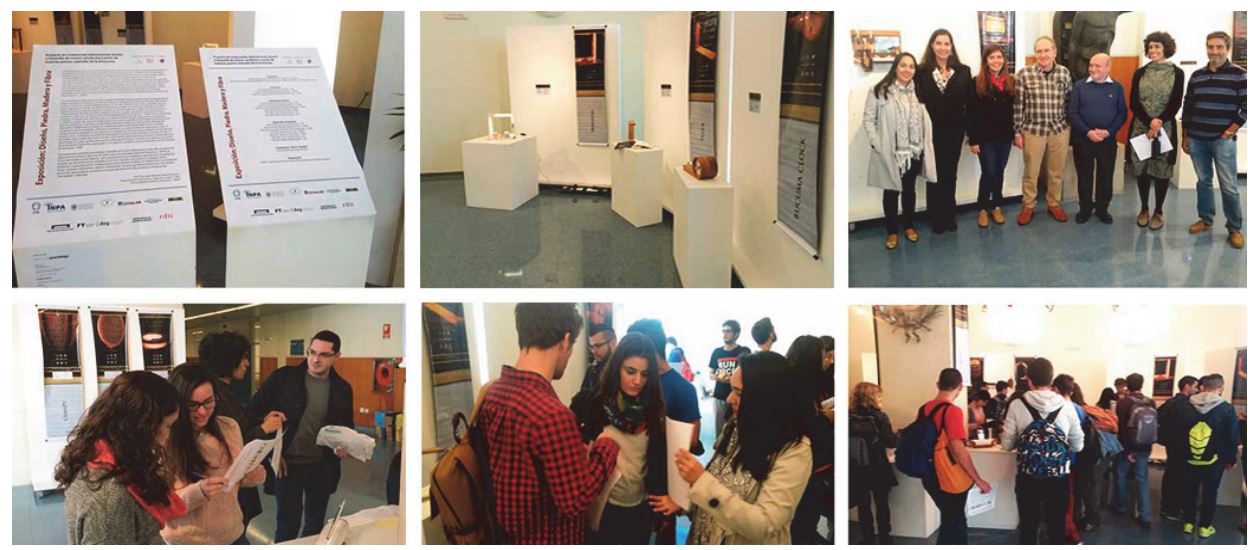

Fig. 18 Exposição dos protótipos na Universitat Politècnica de València (ETSID/UPV - Espanha)

Em ambas as exposições, os participantes foram consultados a respeito da combinação feita entre os materiais utilizados nas peças, os atributos do design dos produtos e os novos produtos projetados a partir do uso da fibra vegetal de tucumã-i (Astrocaryum acaule), da pedra mármore branco de Portugal com veios e de algumas espécies de madeiras naturais da Amazônia. De acordo com os dados registrados e analisados por meio da distribuição de freqüência e o cálculo de médias, a maioria dos entrevistados ( $\mathrm{n}=$ 208; 100\%), aprovou a iniciativa do projeto de combinar três recursos naturais distintos na formação de produtos, pois considera que o uso dessas matérias-primas na composição dos produto oferece boas vantagens com relação a aspectos como: custo/benefício, processo produtivo e benfeitorias aos campos socio-econômico-cultural das localidades envolvidas no projeto. Com respeito aos atributos do design aliados/combinados às características das materias-primas empregadas nos produtos desenvolvidos, numa escala de 1 a 4 - onde 1 significa 'pouco importante' e 4 'bastante importante', o fator 'composição' foi o mais relevante e deve ser o elemento prioritário a ser respeitado $(\mathrm{m}=3,38)$, sendo reforçado pelos fatores de 'resistência' $(\mathrm{m}=3,31)$ e 'valor cultural' $(\mathrm{m}=3,24)$. Para o universo amostral, a reunião desses três aspectos fez das propostas de produtos um diferencial para a segmentação de mercado escolhida pelo projeto, além de oferecer benefícios aos seus possíveis consumidores. Quanto aos novos produtos apresentados nas exposições, grande parte do público consultado $(n=208 ; 93 \%)$, afirmar a ideia transmitida pelo conjuto de objetos criações é a 'inovação', fator considerado, pelo universo amostral, como fundamental aos projetos de design que trabalham com a transferência de conhecimento e de tecnologias para a gestão e fabricação de novos produtos, acompanhadas da valorização dos aspectos técnicos, científicos, socioculturais, econômicos e eco sustentáveis, para o alcance de novos mercados. Após constatar a positiva aceitação por parte de ambas as comunidades acadêmicas, localizadas Manaus/Brasil e Valencia/Espanha, e receber os cumprimentos por parte do Instituto Nacional de Pesquisas da Amazônia (INPA) e do Centro Tecnológico da Pedra Natural de Portugal (CEVALOR), demonstrando a imensa satisfação com os resultados alcançados com a pesquisa, a Universidade Federal do Amazonas - através da equipe técnica do projeto de cooperação internacional: pedra, madeira e fibra, conseguiu estreitar os laços entre as instituições parceiras e receber convites para futuras atividades de pesquisa em conjunto. Além disso, o projeto ainda pode relacionar os seguintes resultados positivos obtidos:

- 13 (treze) protótipos de produtos, confeccionados com duas ou três das matérias-primas de estudo: pedra, madeira e fibra; 
- 1000 (mil) exemplares de Catálogo dos Produtos confeccionados através do projeto, publicação cujo número do ISBN é: 978-85-7401-808-9;

- 13 (treze) Pedidos para Registro de Patentes, dos quais 11 (onze) foram aprovados recebendo aos devidas numerações que podem ser consultadas no banco de dados do Instituto Nacional da Propriedade Industrial (INPI): BR3020150058021 / BR3020150059583 / BR3020150059567 / $\mathrm{BR} 3020150058307$ / BR3020150058315 / BR3020150058331 / BR3020160008795 / BR3020160008809 / BR3020160011397 / BR30 20160011192 / BR3020160011184. Dos demais pedidos encontram-se em processo de avaliação no INPI;

- Apresentação dos produtos do projeto, através de exposições no Brasil e na Espanha;

- Alto grau de aceitação por parte do público visitante de ambas as exposições;

- Quatro artigos publicados em congressos internacionais nos EUA, Brasil e Espanha.

Encerrando as atividades, a coordenação geral do projeto recebeu um convite por parte da Reitora da Universidade Federal do Amazonas, para participar da solenidade de apresentação do catálogo de produtos do projeto de cooperação internacional, junto a outros lançamentos da Editora da UFAM (Fig. 19), como forma de reconhecer a dimensão da pesquisa e a importância do trabalho desenvolvido, bem como a internacionalização da produção científica da instituição. Na ocasião, toda a equipe técnica do projeto foi parabenizada pelo significativo e maravilhoso trabalho realizado em parceria com o Instituto Nacional de Pesquisas da Amazônia (INPA), o Centro Tecnológico da Pedra Natural de Portugal (CEVALOR/Portugal) e a Universitat Politècnica de València (UPV/Espanha).

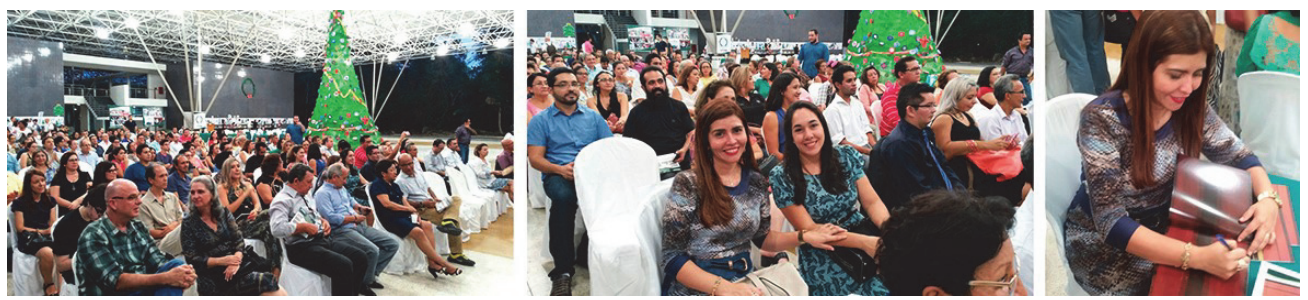

Fig. 19 Coordenação técnica do projeto no lançamento do catálogo dos produtos pela Editora UFAM

Torna-se importante ressaltar que a força de vontade em atingir os objetivos traçados pelo projeto, foi grandiosa por parte dos seus componentes, algo que facilitou significativamente para a realização da fase de levantamento de dados da pesquisa e, principalmente, da produção dos protótipos. As dificuldade foram muitas, contudo foi possível alcançar além das metas atingidas. Através da união e determinação da equipe técnica, realizar muitos feitos, entre deles:

- Capacitação dos discentes de Design do Departamento de Design e Expressão Gráfica da Universidade Federal do Amazonas (DEG/UFAM para o desenvolvimento de novos produtos com matérias-primas naturais da Amazônia;

- Troca de experiência profissional, técnica e científica entre os seus participantes;

- Valorização de matérias-primas naturais regionais;

- Valorização do saber tradicional, cultural e do design; 
- Incentivo a participação de acadêmicos, técnicos e docentes em projetos de pesquisa que tenho o design como fator diferencial e estratégico para o melhor uso e aplicação de recursos naturais no mercado de produtos decorativos e utilitários;

- Interesse pela realização de novos projetos com instituições internacionais.

\section{Referências}

BLAXTER, L.; HUGHES, C. y TIGHT, M. (2002). Cómo se hace una investigación. Barcelona: Gedisa.

CEVALOR. (28 de Novembro de 2014). Equipamentos NEWTECHSTONE Lab. Borba. Fonte: CEVALOR - Centro Tecnológico da Pedra Natural de Portugal. : $<$ http://cevalor.pt/ $>$ [Consulta: 10 de outubro de 2015].

DE TONI, D.; MILAN, G. S. y SCHULER, M. (2005). O desenvolvimento de novos 274roductos: um estudo exploratório ambientado em empresas de acessórios plásticos para móveis. Revista Produção. ISSN 1676 - 1901, Vol.5, Num. 2, Universidade Federal de Santa Catarina Florianópolis - SC - Brasil.

GIL, A. C. (2010). Como elaborar projetos de pesquisa. 5 ed. São Paulo: Atlas.

INETI. (28/11/2014 de Novembro de 2014). Rochas Ornamentais Portuguesas. Fonte: INETI - Instituto Nacional de Engenharia Tecnologia e Inovação I.P.: <http://rop.ineti.pt/rop/FormProduto.php> [Consulta: 12 de março de 2015].

MACIEL, K. M. F. et al (2011). "Análise do posicionamento do Design quanto ao uso semi-industriais da fibra amazôniza de tucumã-i (Astrocaryum acaule) para a fabricação de produtos”. In: CONGRESSO UEM DE DESIGN, Paraná. s.1, s.n, 2011. p. 1-4.

MARCONI, M. A. y LAKATOS, E. M. (2006). Fundamentos de metodologia cientifica. São Paulo: Atlas.

NASCIMENTO, C. C., MONTEIRO DE PAULA, E. V. C. (2012). "A floresta e seus produtos madeireiros". In: HIGUCHI, Inês Gasparetto; HIGUCHI, Niro (edit.). A floresta e suas múltiplas dimensões: uma proposta de educação ambiental. 2. ed. Manaus s.n.: Editora INPA.

PACHECO, K. M. M. et al (2011). "Análise do posicionamento de especialistas sobre o uso da fibra natural amazônica de tucumã-i (Astrocaryum acaule) para o desenvolvimento de produtos semi-industriais". In: Anais do III Simpósio Brasileiro de Design Sustentável (III SBDS) - Proceedings of the 3rd International Symposium on Sustainable Design (ISSD). Editora Universitária UFPE. Pernambuco/Brasil. p. 374-389.

PACHECO, K. M. M.; ORTUÑO, B. H.; MIRANDA, I. P. A.; NASCIMENTO, C. C. Y PACHECO, A. S (2011). "Oportunidades e limitações do uso da fibra natural de tucumã-i (Astrocaryum acaule) para a gestão e desenvolvimento de produtos semi-industriais". Anais do $8^{\circ}$ Congresso Brasileiro de Gestão de Desenvolvimento de Produto (CBGDP). UFRGS/FEEng. Porto Alegre - RS/ Brasil, 2011. p. 9230-9246.

PAIXÃO-BARRADAS, S. (2008). Picnic: A Paisagem e a pedra. Lisboa: Colibri. 